\title{
Neurotoxicidad del metotrexato intratecal relacionado con demencia, ataxia y disfunción del sistema autónomo. Reporte de un caso
}

\author{
José Darío Ochoa-Mena y César Alejandro Arce-Salinas \\ Servicio de Medicina Interna, Hospital Central Sur de Alta Especialidad PEMEX, Ciudad de México, México
}

\begin{abstract}
Resumen
El metotrexato intratecal se emplea en diversas neoplasias hematológicas que involucran al sistema nervioso central. La descripción de la neurotoxicidad es en su mayoría subaguda y reversible, y el espectro clínico es variable, desde déficit neurológico focal hasta encefalopatía progresiva e irreversible. Los cambios en la resonancia magnética son leucoencefalopatía y lesiones en el cerebelo. Se presenta el caso de una mujer de 55 años con linfoma no Hodgkin de células $B$ manejada con esquema R-CHOP (rituximab, ciclofosfamida, clorhidrato de doxorubicina [hidroxidaunorubicina], sulfato de vincristina [oncovin] y prednisona) y metotrexato intratecal por sospecha de infiltración cerebral de la enfermedad, con desarrollo posterior de ataxia, demencia y disautonomía, y neuroimagen con atrofia cerebelosa y del tallo cerebral. La neurotoxicidad por metotrexato afectó a múltiples regiones del encéfalo, causó disfunción grave y se relacionó al cuadro clínico como secuela irreversible.
\end{abstract}

Palabras clave: Atrofia cerebelosa. Metotrexato intratecal. Atrofia de múltiples sistemas. Neurotoxicidad.

\section{Neurotoxicity of intrathecal methotrexate related to dementia, ataxia and autonomic system dysfunction. Case report}

\begin{abstract}
The intrathecal methotrexate is used in various hematological neoplasms that involves the central nervous system. The description of neurotoxicity has been performed predominantly in the pediatric population for decades, with a clinical spectrum mostly subacute and reversible, since focal neurological deficit until progressive and irreversible encephalopathy. The changes in magnetic resonance in most reports have been leukoencephalopathy and occasionally cerebellar lesions have been described, however there are no reports of brainstem and cerebellum atrophy. We present the case of a 55-year-old woman who was diagnosed with diffuse B-cell non-Hodgkin lymphoma treated with intravenous rituximab, cyclophosphamide, doxorubicin, vincristine and prednisone and intrathecal methotrexate chemotherapy cycles at recurrent doses due to suspicion of cerebral infiltration of the disease, with subsequent clinical symptoms an radiologic findings similar to brainstem and cerebellum atrophy. Methotrexate neurotoxicity affected multiple regions of the brain causing atrophy and was related to the clinical syndrome as irreversible sequelae.
\end{abstract}

Key words: Cerebellum atrophy. Intrathecal methotrexate. Brainstem atrophy. Neurotoxicity.

\section{Correspondencia:}

José Darío Ochoa-Mena

Av. Periférico Sur, 4091

Col. Fuentes del Pedregal, Del. Tlalpan Fecha de recepción: 13/03/2018

C.P. 14140, Ciudad de México, México Fecha de aceptación: 17/08/2018

E-mail: dario.ochoa.mena@gmail.com_DOI: 10.24875/RMN.M18000010

Disponible en internet: 13-11-2018 Rev Mex Neuroci. 2018;19(5):68-72 (http://creativecommons.org/licenses/by-nc-nd/4.0/). 


\section{Introducción}

El metotrexato intratecal es una importante opción en el manejo de la infiltración cerebral del linfoma no Hodgkin; sin embargo, también se han reportado complicaciones devastadoras permanentes e incluso la muerte relacionadas con su uso ${ }^{1}$. Los hallazgos reportados en resonancia magnética se han descrito como cambios en la sustancia blanca en la gran mayoría de las veces ${ }^{2}$. Al parecer, la neurotoxicidad se produce por múltiples factores, y la apoptosis juega un papel importante en su patogénesis ${ }^{3}$. Las manifestaciones clínicas son variadas, desde síndrome tóxico agudo hasta síntomas y signos que mimetizan diversas entidades neurológicas definidas ${ }^{4}$. En el presente trabajo reportamos un caso de neurotoxicidad por metotrexato intratecal con cuadro clínico de ataxia, demencia y disfunción del sistema autónomo.

\section{Reporte de un caso}

Mujer de 55 años con historia de linfoma no Hodgkin en remisión. Cuatro meses atrás presentó debilidad de los miembros inferiores, que le condicionó dificultad para subir escaleras y torpeza para dar pasos, lo cual la obligó a permanecer sentada o en cama; progresó en una semana. A ello se agregó cefalea holocraneana pulsátil -de intensidad 8/10 evaluada con la escala visual análoga- y desorientación. Cuando la paciente fue hospitalizada, estaba desorientada en tiempo y lugar, y presentaba disminución de la fuerza distal y proximal de 4-/5 grados de la escala analítica de fuerza muscular propuesta por Daniels, Williams y Worthingham. Se le realizó punción lumbar, la cual mostró: leucocitos, $60 \mathrm{~mm}^{3}$; proteínas, $120 \mathrm{mg}$; tinciones negativas (tinta china y Gram); PCR para herpes de tipo 1 y 2; Epstein Barr, zóster y enterovirus negativos. En la tomografía de cráneo no se observaron cambios de densidad, lesiones ni alteraciones cualitativas del volumen cerebral (Fig. 1). Los exámenes de laboratorio mostraron: leucocitos, $4.5 \times 10^{3} / \mathrm{ul}$; neutrófilos, $3.8 \times$ $10^{3} / \mathrm{ul} ; \mathrm{Hb}, 14.2 \mathrm{~g} / \mathrm{dl}$; plaquetas, $128 \times 10^{3} / \mathrm{ul}$; glucosa, $111 \mathrm{mg} / \mathrm{dl}$; urea, $40.7 \mathrm{mg} / \mathrm{dl}$; $\mathrm{Cr}, 1.15 \mathrm{mg} / \mathrm{dl}$; sodio, 140 $\mathrm{mmol} / \mathrm{l}$, y potasio, $5.5 \mathrm{mmol} / \mathrm{l}$. Por sospecha de infiltración al SNC, se decidió aplicar $12 \mathrm{mg}$ de metotrexato intratecal por semana hasta completar cuatro dosis. La paciente tuvo mejor orientación y mejoró la marcha y la fuerza $-4+/ 5$ distal y $5 / 5$ proximal bilateral-, por lo que fue dada de alta.

Sin embargo, 14 días después presentó dificultad para recordar el camino a su casa, cómo utilizar los

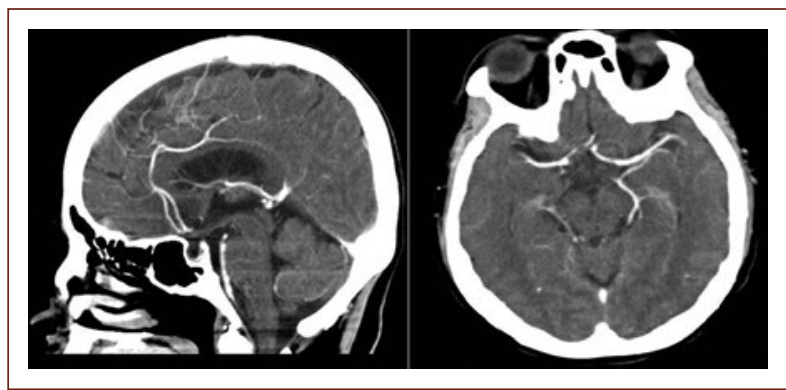

Figura 1. Tomografía con contraste aparentemente normal, sin evidencia de atrofia de valoración de forma cualitativa.

cubiertos, dificultad para nominar objetos, lenguaje escandido e imposibilidad para sumar o restar. En consecuencia, fue hospitalizada de nuevo, y durante su estancia presentó nistagmo inagotable y ataxia de las cuatro extremidades, que progresó en los tres meses posteriores a incontinencia de esfínteres. Se registraron eventos repetidos de fluctuaciones en la presión arterial sistólica $>20 \mathrm{mmHg}$ y en la diastólica $>10 \mathrm{mmHg}$, sin evidenciar aumento de la frecuencia cardíaca de más de 30 latidos por minuto acompañado de diaforesis y ortostatismo con lipotimia con los cambios de posición de decúbito supino a bipedestación. La paciente fue referida a un centro de tercer nivel de atención, en donde dos días después progresó a estupor con alteración de la mecánica ventilatoria, por lo que requirió ventilación mecánica invasiva con manejo en terapia intensiva y permanencia durante dos meses hasta la retirada del ventilador. Una vez estabilizada, reingresó a planta de hospitalización y se realizaron los siguientes estudios: punción lumbar -la cual evidenció leucocitos de $3 / \mathrm{mm}^{3}$ y proteínas de $46 \mathrm{mg} / \mathrm{dl}$-, tinciones negativas (tinta china y Gram) e imagen por resonancia magnética, en la que se observó atrofia del tallo cerebral y cerebeloso, con lesiones hiperintensas en cerebelo y subcorticales en el lóbulo temporal bilateral (Figs. 2-5). La paciente persistió con ataxia de las cuatro extremidades, nistagmo inagotable, imposibilidad para hablar, indiferencia al medio, incontinencia de esfínteres, rigidez de las cuatro extremidades, las mismas fluctuaciones en la tensión arterial, con diaforesis, sin evidenciar causa infecciosa o metabólica, hasta su egreso seis meses después del inicio de su sintomatología. Falleció nueve meses después del inicio de su padecimiento debido a complicaciones infecciosas como neumonía.

A continuación se presenta una breve revisión de la literatura médica respecto al uso del metotrexato intratecal. 


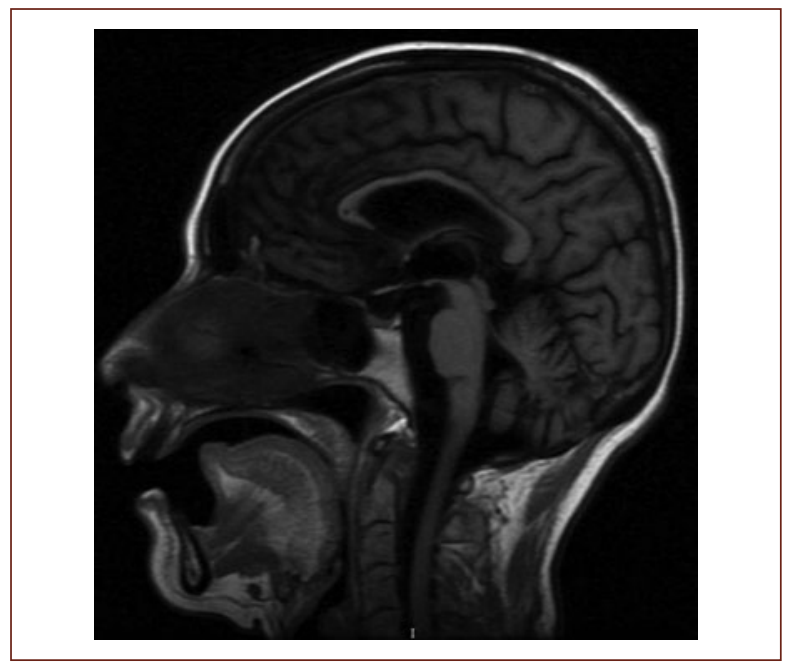

Figura 2. Corte sagital en secuencia T1 que demuestra la atrofia del tallo cerebral y cerebelo de forma cualitativa, incluyendo cuerpo calloso.

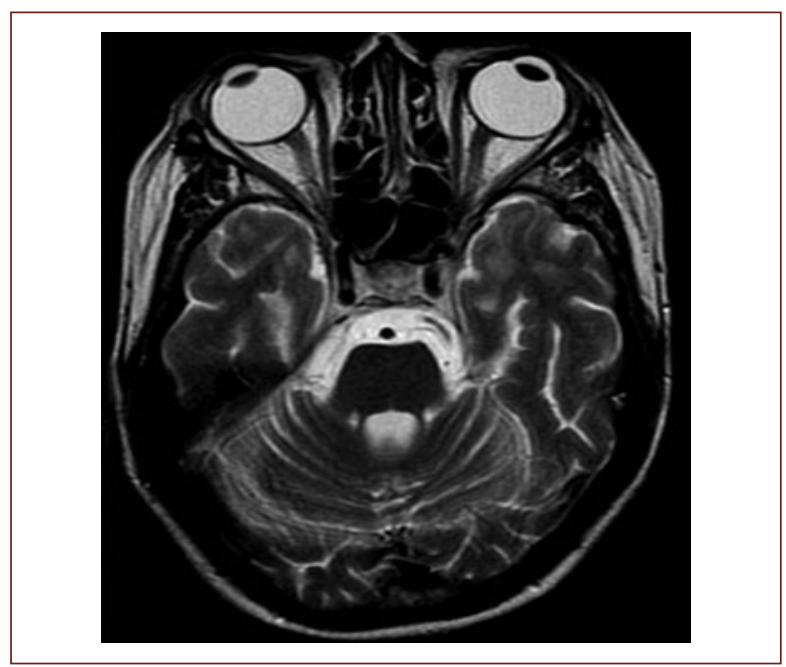

Figura 3. Lesiones subcorticales de regiones temporales, además de la atrofia cualitativa a nivel del puente. Secuencia T2, corte axial.

\section{Metotrexato intratecal}

El metotrexato se utiliza en el tratamiento de la leucemia, linfoma y otros tumores sólidos. Su mecanismo de acción consiste en inhibir la enzima dihidrofolato reductasa, lo que ocasiona una depleción de folato que provoca una incapacidad celular para sintetizar purinas y timidina, interrumpiéndose así la síntesis de ADN y $\mathrm{ARN}^{5}$. El metotrexato puede producir toxicidad en el sistema nervioso central en forma aguda (primeras $24 \mathrm{~h}$ ), subaguda (10 días después) o tardía (semanas a meses). Las alteraciones suelen ser reversibles, pero se

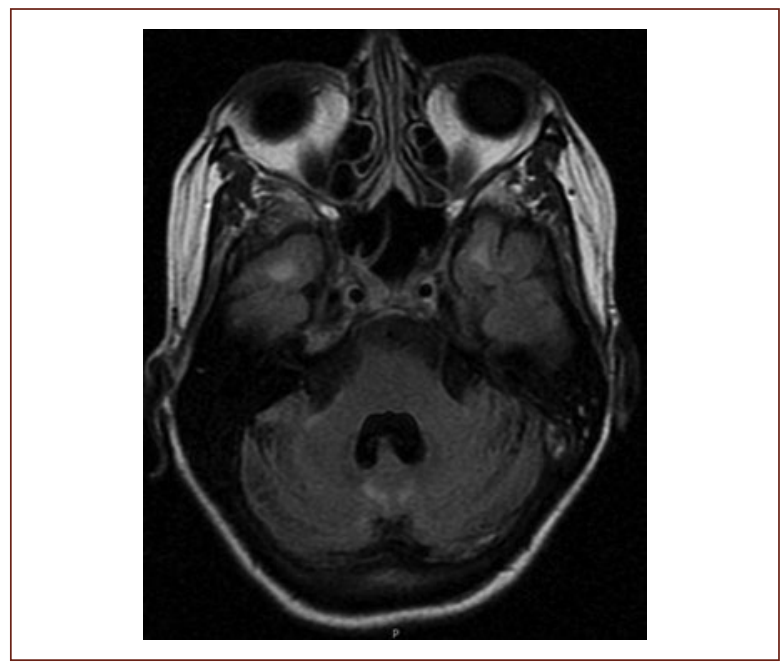

Figura 4. Lesiones hiperintensas en cerebelo y regiones temporales en FLAIR.

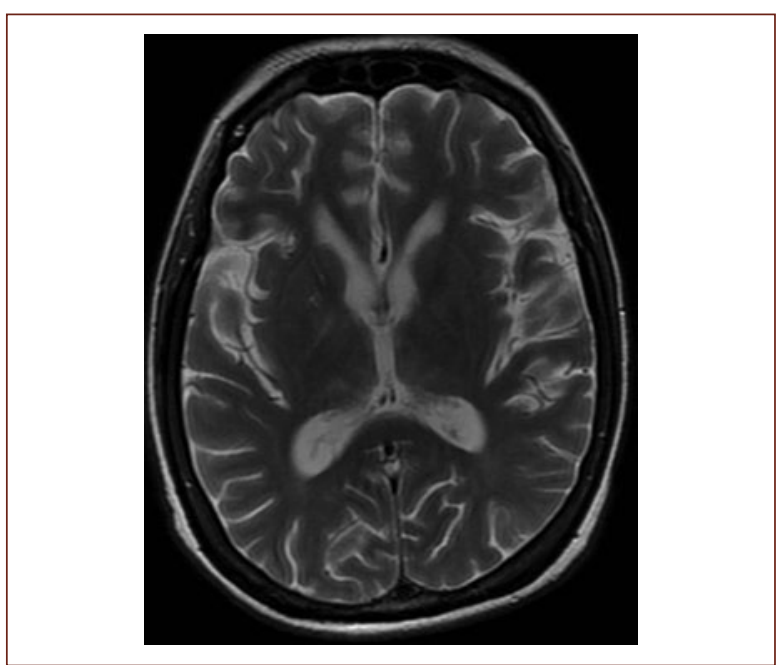

Figura 5. Hiperintensidad de ganglios basales, incluyendo putamen bilateral.

han descrito casos de neurotoxicidad con secuelas neurológicas permanentes o de evolución fatal ${ }^{6}$.

\section{Efectos adversos}

Alrededor del 3-4\% de quienes reciben metotrexato intratecal desarrollarán neurotoxicidad. De forma aguda, el $61 \%$ puede presentar síndrome tóxico caracterizado por fiebre, cefalea y vómitos; otros efectos adversos más raros en esta fase son convulsiones, hemorragia parenquimatosa y paraplejía ${ }^{7}$. La mayoría de las manifestaciones se presentan después de 7-14 
Tabla 1. Resumen de las manifestaciones descritas por neurotoxicidad debido al uso de metotrexato intratecal

\begin{tabular}{|c|c|c|}
\hline Autor & Año & Manifestación clínica \\
\hline Bleyer & 1973 & Mielopatía \\
\hline Gagliano & 1976 & Paraplejía aguda \\
\hline Shapiro ${ }^{19}$ & 1984 & Meningitis aséptica subaguda y encefalopatía fatal \\
\hline Gilbert & 1989 & Desmielinización por disfunción de oligodendrocitos \\
\hline Kubo ${ }^{20}$ & 1992 & Encefalopatía transitoria \\
\hline Mahoney ${ }^{21}$ & 1998 & Síndrome tóxico con convulsiones \\
\hline Antunes ${ }^{22}$ & 2002 & Síndrome de Klüver-Bucy y crisis uncinadas \\
\hline Fliessbach $^{23}$ & 2003 & Deterioro cognitivo y demencia \\
\hline Brock & 2004 & Encefalomielitis fatal aguda \\
\hline Boran $^{24}$ & 2008 & Hematoma intraparenquimatoso \\
\hline Masterson ${ }^{25}$ & 2009 & Atrofia cerebelar \\
\hline Hamamoto $^{26}$ & 2009 & Esclerosis mesial temporal \\
\hline Tufekci ${ }^{27}$ & 2011 & Diplopía, disfagia, déficits hemisensoriales, hemiparesia subaguda \\
\hline Yap 28 & 2016 & Hemiparesia alternante y apraxia orolingual \\
\hline Schmidt & 2017 & Presencia en electroencefalograma de Delta Brush extremos \\
\hline Deneux ${ }^{29}$ & 2017 & Síndrome pseudoinfarto (hemiparesia, déficit hemisensorial alternante, afasia) agudo \\
\hline
\end{tabular}

días en forma subaguda con espectro clínico amplio, desde déficit focal hasta encefalopatía, incluyendo la posterior reversible ${ }^{8}$; otras formas más raras, como hemiparesia alternante con apraxia orolingual y síndrome de cauda equina, también se han descrito ${ }^{9}$. De forma crónica se ha reportado alteración de la marcha, incontinencia urinaria, demencia y muerte por edema cerebral fulminante ${ }^{10}$.

\section{Neurotoxicidad}

La toxicidad no se pude explicar por un único factor. Recientemente se ha medido la actividad oxidante con incremento progresivo de caspasa $3 / 7$ e isoprostano F2, los cuales incrementan la oxidación de los fosfolípidos, lo que ocasiona muerte celular. En estudios de genoma-wide se ha intentado asociar polimorfismos como GSTP1, MTHFR y SHMT1 que dan susceptibilidad a leucoencefalopatía y neurotoxicidad por metotrexato ${ }^{3,11}$. También se ha sugerido la participación del daño axonal directo y la desmielinización, las alteraciones vasculares, el déficit de folato y los desequilibrios de la adenosina y la homocisteína como factores participantes ${ }^{12}$.

\section{Discusión}

Entre los diferentes reportes de los síntomas por neurotoxicidad del metotrexato intratecal, la atrofia del tallo cerebral y cerebelo presentada de forma aislada en este caso forma parte de los efectos adversos devastadores y permanentes, hasta ahora no descrita, como se resume en la tabla 1.

En 1973, W.A. Bleyer describió daños severos como mielopatía cuando los niveles de metotrexato en el líquido cefalorraquídeo eran 13 veces más elevados que la media $\left(1.7 \times 10^{-7}\right)^{13,14}$. A la paciente se le aplicaron cuatro dosis de $12 \mathrm{mg} / \mathrm{m}^{2}$, que no exceden más de $100 \mathrm{mg}$, lo cual hace suponer que la susceptibilidad era mayor.

Se han descrito reportes de paraplejía posterior a las $6 \mathrm{~h}$ del uso intratecal como reversibles, aunque en algunos casos también como permanentes ${ }^{15}$. La encefalopatía fatal es una complicación rara y se ha descrito como subaguda ${ }^{16}$. La disfunción autonómica, cerebelosa y cognitiva de este caso se presentó como un cuadro progresivo e irreversible, considerándose en un principio subaguda, aunque progresó a crónica y permanente.

Dentro de la fisiopatología, el daño axonal y desmielinizante se debe en un primer momento a la disfunción 
de oligodendrocitos ${ }^{17}$. En el caso presentado la resonancia mostró cambios desmielinizantes subcorticales compatibles con la lesión a la sustancia blanca. Asimismo, la apoptosis que origina el metotrexato intratecal sugiere que ocasionó la atrofia a nivel del tallo cerebral y diencéfalo de la paciente.

En el electroencefalograma se ha evidenciado la presencia de descargas laterales epileptiformes periódicas durante la encefalopatía transitoria ocasionada por el uso de metotrexato intratecal, así como también se han descrito los Delta Brush extremos ${ }^{18}$. En cambio, el EEG del caso presentado sólo mostró lentitud generalizada, compatible con la disfunción global.

Los mecanismos involucrados en la neurotoxicidad son variados. Recientemente se ha probado el uso de dextrometorfano previo al uso del metotrexato intratecal debido al involucramiento de los receptores NMDA en la neurotoxicidad. El uso de ácido folínico a dosis de $8 \mathrm{mg} / \mathrm{m}^{2}$ o de $100-500 \mathrm{mg}$ intravenoso ha tenido resultados variables, ya sea sólo o en combinación con esteroides a altas dosis ${ }^{19}$. En nuestro caso, a pesar del manejo con esteroides la paciente no tuvo mejoría alguna.

La presentación clínica de hipotensión fluctuante, síndrome cerebeloso y demencia con datos de rigidez se estableció de forma permanente en la paciente. Estos signos y síntomas, además de los cambios en la resonancia magnética con atrofia del tallo cerebral y cerebelo e hipointensidades en T2 a nivel del putamen, explicaron la clínica irreversible de este caso.

\section{Conclusiones}

El metotrexato intratecal tiene consecuencias fatales e incapacitantes, ya sea de forma aguda, subaguda 0 crónica. Afecta al sistema nervioso central de formas variadas, simulando síndromes clínicos y radiológicos definidos en los diagnósticos diferenciales neurológicos. Tal es el caso presentado, con clínica de demencia, disfunción autonómica y ataxia, que se correlacionaron en la resonancia magnética. Consideramos que razonablemente se descartaron otras entidades clínico-radiológicas que podrían explicar el cuadro de la paciente, aunque desconocemos si existen otros factores propios del huésped que predisponen a neurotoxicidad de la magnitud descrita en nuestro caso.

\section{Bibliografía}

1. García Hernández I, Rey Galán C, Concha Torre A, Los Arcos Solas M, Mayordomo Colunga J. Neurotoxicidad subaguda letal por metotrexato intratecal. Bol Pediatr. 2012;52(219):37-42.

2. Brugnoletti F, Morris EB, Laningham FH, Patay Z, Pauley JL, Pui CH, et al. Recurrent intrathecal methotrexate induced neurotoxicity in an adolescent with acute lymphoblastic leukemia: serial clinical and radiologic findings. Pediatr Blood Cancer. 2009;52(2):293-5.

3. Taylor OA, Hockenberry MJ, McCarthy K, Gundy P, Montgomery D, Ross A, et al. Evaluation of biomarkers of oxidative stress and apoptosis in patients with severe methotrexate neurotoxicity: a case series. J Pediatr Oncol Nurs. 2015;32(5):320-5.

4. Geiser CF, Bishop Y, Jaffe N, Furman L, Traggis D. Adverse effects of intrathecal methotrexate in children with acute leukemia in remission. Blood. 1975;45(2):189-95.

5. Duttera JM, Gallelli JF, Kleinman LM, Tangrea JA, Wittgrove AC. Intrathecal methotrexate. Lancet. 1972;1(7749):540.

6. Bleyer WA. Clinical pharmacology of intrathecal methotrexate. II. An improved dosage regimenderived from age-related pharmacokinetics. Cancer Treat Rep. 1977;61(8):1419-25.

7. García-Puig M, Fons-Estupiñá MC, Rives-Solà S, Berrueco-Moreno R, Cruz-Martínez O, Campistol J. Neurotoxicidad por metotrexato en pacientes pediátricos. Descripción de síntomas clínicos y hallazgos radiológicos. Rev Neurol. 2012;54(12):712-8.

8. Dicuonzo F, Salvati A, Palma M, Lefons V, Lasalandra G, De LF, et al. Posterior reversible encephalopathy syndrome associatedwith methotrexate neurotoxicity: conventional magnetic resonanceand diffusion-weighted imaging findings. J Child Neurol. 2009;24:1013-8.

9. Park S, Kang Jl, Bang H, Kim BR, Lee J. A case of the cauda equina syndrome associated with the intrathecal chemotherapy in a patient with primary central nervous system lymphoma. Ann Rehabil Med. 2013:37(3):420-5.

10. Weigel R, Senn P, Weis J, Krauss JK. Severe complications after intrathecal methotrexate (MTX) for treatment of primary central nervous system lymphoma (PCNSL). Clin Neurol Neurosurg. 2004;106(2):82-7.

11. Bhojwani D, Sabin ND, Pei D, Yang JJ, Khan RB, Panetta JC, et al. Methotrexate-induced neurotoxicity and leukoencephalopathy in childhood acute lymphoblastic leukemia. J Clin Oncol. 2014;32(9):949-59.

12. Vezmar S, Becker A, Bode U, Jaehde U. Biochemical and clinical aspects of methotrexate neurotoxicity. Chemotherapy. 2003;49(1-2):92-104.

13. Bleyer WA, Drake JC, Chabner BA. Neurotoxicity and elevated cerebrospinal-fluid methotrexate concentration in meningeal leukemia. $\mathrm{N}$ Engl $\mathrm{J}$ Med. 1973;289(15):770-3.

14. Gagliano RG, Costanzi JJ. Paraplegia following intrathecal methotrexate: report of a case and review of the literature. Cancer. 1976;37(4):1663-8.

15. Brock $S$, Jennings HR. Fatal acute encephalomyelitis after a single dose of intrathecal methotrexate. Pharmacotherapy. 2004;24(5):673-6.

16. Gilbert MR, Harding BL, Grossman SA. Methotrexate neurotoxicity: in vitro studies using cerebellar explants from rats. Cancer Res. 1989; 49(9):2502-5.

17. Schmidt LS, Kjær TW, Schmiegelow K, Born AP. EEG with extreme delta brush in young female with methotrexate neurotoxicity supports NMDA receptor involvement. Eur J Paediatr Neurol. 2017;21(5):795-7.

18. Afshar M, Birnbaum D, Golden C. Review of dextromethorphan administration in 18 patients with subacute methotrexate central nervous system toxicity. Pediatr Neurol. 2014;50(6):625-9.

19. Shapiro WR, Young DF. Neurological complications of antineoplastic therapy. Acta Neurol Scand Suppl. 1984;100:125-32.

20. Kubo M, Azuma E, Arai S, Komada Y, Ito M, Sakurai M. Transient encephalopathy following a single exposure of high-dose methotrexate in a child with acute lymphoblastic leukemia. Pediatr Hematol Oncol. 1992;9(2):157-65.

21. Mahoney DH Jr, Shuster JJ, Nitschke R, Lauer SJ, Steuber CP, Winick N, et al. Acute neurotoxicity in children with B-precursor acute lymphoid leukemia: an association with intermediate-dose intravenous methotrexate and intrathecal triple therapy--a Pediatric Oncology Group study. 1998;16(5):1712-22.

22. Antunes NL, Souweidane MM, Lis E, Rosenblum MK, Steinherz PG Methotrexate leukoencephalopathy presenting as Klüver-Bucy syndrome and uncinate seizures. Pediatr Neurol. 2002;26(4):305-8.

23. Fliessbach K, Urbach H, Helmstaedter C, Pels H, Glasmacher A, Kraus JA, et al. Cognitive performance and magnetic resonance imaging findings after high-dose systemic and intraventricular chemotherapy for primary central nervous system lymphoma. Arch Neurol. 2003;60(4):563-8.

24. Boran P, Tokuc G, Boran BO, Oktem S. Intracerebral hematoma as a complication of intrathecal methotrexate administration. Pediatr Blood Cancer. 2008;50(1):152-4.

25. Masterson K, Merlini L, Lövblad KO. Coexistence of reversible cerebral neurotoxicity and irreversible cerebellar atrophy following an intrathecal methotrexate chemotherapy: two case reports. J Neuroradiol. 2009;36(2):112-4.

26. Hamamoto K, Oriuchi N, Kanazawa T, Higuchi T, Endo K. Mesial temporal sclerosis associated with methotrexate-induced leukoencephalopathy. Pediatr Neurol. 2009:40(4):306-9.

27. Tufekci O, Yilmaz S, Karapinar TH, Gozmen S, Cakmakci H, Hiz S, et al. A rare complication of intrathecal methotrexate in a child with acute lymphoblastic leukemia. Pediatr Hematol Oncol. 2011;28(6):517-22.

28. Yap SM, MacEneaney P, Ryan C, O'Toole O. Alternating hemiparesis and orolingual apraxia as manifestations of methotrexate neurotoxicityin a paediatric case of acute lymphoblastic leukaemia. BMJ Case Rep. 2016;2016.

29. Deneux V, Leboucq N, Saumet L, Haouy S, Akbaraly T, Sirvent N. [Acute methotrexate-related neurotoxicity and pseudo-stroke syndrome]. Arch Pediatr. 2017;24(12):1244-8 